ËESTI NSV TEADUSTE AKADEEMIA TOIMETISED.

FOOSIKA * MATEMAATIKA

ИЗВЕСТИЯ АКАДЕМИИ НАУК ЭСТОНСКОГ ССР. ФИЗИКА * МАТЕМАТИКА PROCEEDINGS OF THE ACADEMY OF SCIENCES OF THE ESTONIAN SSR. PHYSICS * MATHEMATICS

1986, 35, 4

УдК $62-501.7$

Н. РАНДВЕЭ

\title{
АЛГОРИТМ ВЫБОРА ПРЕДСТАВЛЕНИЯ ОПТИМАЛЬНОГО УПРАВЛЕНИЯ ЛИДЕРА
}

I. RANDVEE. ALGORITM OPTIMAALSE JUHTTOIME ESITUSVIISI VALIKUKS

I. RANDVEE. AN INCENTIVE SCHEME FOR LINEAR QUADRATIC SYSTEMS

$$
\text { (Представил Н. Алумяэ) }
$$

Известно $\left[{ }^{1,2}\right]$, что при управлении динамическими системами, состоящими из нескольких (независимых) центров, приоритетный центр (лидер) может уетановить в системе режим, который соответствует глобальному минимуму своей функции цели. Ниже приводится алгоритм выбора представления закона управления, с помощью которого лидер может воздействовать на функцию цели последующего центра и реализовать в линейной дискретно-непрерывной системе желаемую ему допустимую траекторию движения.

1. Формальное описание задачи

Дана динамическая система

$$
\dot{x}=f\left(x, u_{1}, u_{2}, t\right), \quad x \in R^{n}, \quad u_{i} \in R^{r_{t}},
$$

которая управляется из двух центров. Приоритетный центр использует управление $u_{1}$. Предполагается, что при любой паре допустимых законов управления $u_{i}=p_{i}\left(x(s), \quad t_{0} \leqslant s \leqslant t\right), p_{i} \in \Gamma_{i}$, т. е. для $\left(p_{1}, p_{2}\right) \in$ $\in \Gamma_{1} \times \Gamma_{2}$ при заданном $x_{0}$ существует единственное решение уравнения (1) и конечное значение критерия оптимальности неприоритетного центра $J_{2}\left(x_{0}, p_{1}, p_{2}\right)$. Пусть лидер выбрал желаемое ему решение уравнения (1) - траекторию $\bar{x}(t)$ и соответствующие этому решению законы управления $\left(p_{1}^{0}, p_{2}{ }^{0}\right)$.

Дефинируем множество эквивалентных относительно траектории $\bar{x}(t)$ представлений закона $p_{1}^{0}$ :

$$
\bar{\Gamma}_{1} \triangleq\left\{p_{1} \in \Gamma_{1} \mid p_{1}(\bar{x}, t)=p_{1}^{0}(\bar{x}, t), t_{0} \leqslant t \leqslant t_{f}\right\} .
$$

Задача теперь состоит в выборе такого $p_{1}{ }^{*} \in \Gamma_{1}$, при котором имеет место равенство

$$
p_{2}^{*}(\tilde{x})=p_{2}^{0}(\bar{x}),
$$

где $p_{2}^{*}=\underset{\Gamma_{2}}{\arg \min } J_{2}\left(p_{1}^{*}, p_{2}\right)$,

а $\tilde{x}(t)$ - соответствующее паре $\left(p_{1}{ }^{*}, p_{2}{ }^{*}\right)$ решение уравнения (1). При $\widetilde{x}\left(t_{0}\right)=\bar{x}\left(t_{0}\right)$ и выполнении условия $(2)$, очевидно, траектории $\tilde{x}(t)$ и $\bar{x}(t)$ идентичны. 
2. Алгоритм для дискретно-непрерывных систем

Будем считать, что желаемая для лидера траектория $\bar{x}_{k}(k=0,1, \ldots$ ...N-1) системы

$$
x_{k+1}=A x_{k}+B_{1} u_{k}+B_{2} v_{k}
$$

достигается при линейных законах управления: $u_{k}{ }^{0}=-K_{h} \bar{x}_{k}$ и $v_{k}{ }^{0}=$ $=-L_{k} \bar{x}_{k}$, где $\bar{x}_{k} \in R^{n}, \quad u_{k} \in R^{r_{1}}, \quad v_{k} \in R^{r_{2}}, \quad A, B_{1}, B_{2}, K_{k}, L_{k}$ - известные матрицы.

Определим множество эквивалентных управлений лидера, используя одношаговый прогноз

$$
\bar{\Gamma}_{1, k}=\left\{p_{1, k}\left(x_{k}, x_{k-1}\right)=-K_{k} x_{k}+P_{k}\left(x_{k}-A_{k} x_{k-1}\right)\right\},
$$

где $A_{k}=A-B_{1} K_{k-1}-B_{2} L_{k-1}$, а $P_{k} \in R^{n \times r_{1}} \quad$ неизвестная матрица.

Требуется определить последовательность $P_{k}{ }^{*}, k=0,1, \ldots N-1$, при которой минимум функции цели неприоритетного центра

$$
J_{2}=x_{N}^{\prime} Q_{2 N} x_{N}+\sum_{k=0}^{N-1}\left(x_{k}^{\prime} Q_{2} x_{k}+p_{1, k}^{\prime} R_{21} p_{1, k}+v_{k}^{\prime} R_{22} v_{k}\right)
$$

по управлениям $v_{k}$ достигается при $v_{k}{ }^{*}$ равном $v_{k}{ }^{0}$, т. е. $v_{k}{ }^{*}=-L_{k} \bar{x}_{k}$ для всех $k$.

Минимизация (4) должна осуществляться при условии (3), в котором $u_{k}$ заменен, как и в $(4)$, на $p_{1, k}\left(x_{k}, x_{k-1}\right) \in \bar{\Gamma}_{1, k} ; \quad$ предполагается также, что $Q_{2 N} \geqslant 0, Q_{2} \geqslant 0, R_{21}>0, R_{22}>0$ - известные симметричные матрицы.

В данном случае вторая вариация $J_{2}$ включает $P_{k}$, т. е. у лидера в действительности имеется возможность изменять $J_{2}$, не изменяя при этом $u_{k}{ }^{0}-$ желаемых значений своего сигнала управления.

Задача (3)-(4) решается по схеме синтеза линейных регуляторов с учетом воздействий предшествующей системы $\left[{ }^{3}\right]$. Приоритет лидера при этом обеспечивается исключением управления $v_{k}$ на последнем шаге, т. е. выбором $B_{2}(N)=0$ или $R_{22}(N)=0$.

Искомое $P_{k}{ }^{*}$ определяется на каждом шаге $N, N-1, \ldots, 0$ решением линейного матричного уравнения

$$
B_{2}^{\prime}\left(P^{\prime}{ }_{k} G_{k}+S_{k}\right) A_{k-1}-R_{22} L_{k-1}=0
$$

совместно с рекурсивными уравнениями для матриц $S_{k}$ и $G_{k}$ :

$$
\begin{array}{ll}
S_{k}=Q_{2}+A^{\prime}{ }_{k} S_{k+1} A_{k}+K^{\prime}{ }_{k} R_{21} K_{k}+L^{\prime}{ }_{k} R_{22} L_{k}, & S_{N}=Q_{2 N}, \\
G_{k}=B^{\prime}{ }_{1} P_{k+1} G_{k+1} A_{k}-R_{21} K_{k}+B^{\prime}{ }_{1} S_{k+1} A_{k}, & G_{N}=0 .
\end{array}
$$

Уравнение (5) является необходимым условием минимума функции цели неприоритетного центра на $k$-м шаге. Его решение $P_{k}{ }^{*}$, если оно существует, обеспечивает равенство $v_{k}{ }^{*}$ с желаемым управлением $v_{k}{ }^{0}=$ $=-L_{k} \bar{x}_{k}$.

Ниже в качестве иллюстрации приведены практически установившиеся значения $P_{k}{ }^{*}$ представления закона управления лидера $u_{k}=$ $=-K_{k} x_{k}+P_{k}{ }^{*}\left(x_{k}-A_{k} x_{k-1}\right)$ для шести различных желаемых траекторий скалярной системы

$$
x_{k+1}=0,9 x_{k}+u_{k}+v_{k}, \quad x_{0}=30,
$$

в случае функции цели неприоритетного центра

$$
J_{2}=\sum_{k=0}^{30} x_{k}^{2}+u_{k}^{2}+3 v_{k}^{2}
$$




\begin{tabular}{l|l|l|l|l|l|l} 
& 1 & \multicolumn{1}{|c|}{2} & 3 & \multicolumn{1}{|c|}{4} & \multicolumn{1}{|c|}{5} & \multicolumn{1}{|c|}{6} \\
\hline$K_{15}$ & 0,2 & 0,2 & 0,2 & 0,2 & 0,2 & 0,2 \\
$L_{15}$ & 0,25 & 0,30 & 0,35 & 0,40 & 0,45 & 0,50 \\
$P_{15}$ & 0,2 & 1,0 & 1,6 & 2,3 & 3,2 & 4,3
\end{tabular}

Использование эквивалентных представлений закона управления лидера в виде $p_{k}\left(x_{k}, x_{k-1}\right)$ открывает некоторые новые возможности в задачах управления многоуровневыми системами и системами с двумя шкалами времени $\left[{ }^{4,5}\right]$.

\section{ЛИТЕРАТУРА}

1. Ishida, T., Shimemura, E. Int. J. Control, 38, № 6, 1133-1148 (1983).

2. Zheng, Y.-P., Basar, T., Cruz, J. B. Jr. IEEE Trans. on Systems, Man, and Cybernetics, SNC-14, № 1 (1984).

3. Рандвеэ Н. Изв. АН ЭССР. Физ. Матем., 35, № 1, 107-109 (1986).

4. Рандвеэ И. Изв. АН ЭССР. Физ. Матем., 30, № 1, 35-46 (1981).

5. Salman, M. A., Cruz, J. B. Jr. Int. J. Control, 37, № 6, 1401-1416 (1983).

Институт кибернетики Академии наук Эстонской ССР
Поступила в редакцию $21 /$ II 1986 